\title{
Early awake prone position combined with high-flow nasal oxygen therapy in severe COVID-19: a case series
}

\author{
Qiancheng $\mathrm{Xu}^{1+}$, Tao Wang ${ }^{1+}$, Xuemei Qin ${ }^{1+}$, Yanli $\mathrm{Jie}^{2}$, Lei Zha ${ }^{3}$ and Weihua Lu ${ }^{1 *} \mathbb{D}$
}

Keywords: COVID-19, High-flow nasal cannula, Prone positioning, SARS-CoV-2

Coronavirus disease 2019 (COVID-19) has rapidly spread around the world in the past 3 months and has now become a global public health crisis. The mortality of COVID-19 in some European cities exceeds 11\%, and the fatality rate is up to $61.5 \%$ in critical patients, especially in mechanically ventilated patients [1]. Once mild to moderate patients progress to critical illness, the incidence of septic shock, intubation, and myocardial injury increases significantly. Mechanical ventilation patients need more sedative, analgesic, and neuromuscular blocker drugs, which will affect the patient's hemodynamic status and airway expectoration ability [2]. Previous studies have confirmed that high-flow nasal cannula (HFNC) can reduce the endotracheal intubation rate and mortality in patients with respiratory failure [3]. However, this therapy of COVID-19 cannot improve the pathophysiology of ventilation-perfusion defects and atelectasis, which can be proved by autopsies, i.e., small airways are blocked by mucus plugs [4]. Awake prone position could improve the mismatch of ventilation-perfusion and open the atelectatic lungs by adequate sputum drainage. So far, the role of early awake prone position (PP) combined with HFNC therapy in the treatment of severe COVID-19 has not been reported. So, we conducted a retrospective

\footnotetext{
* Correspondence: Iwh683@126.com

${ }^{\dagger}$ Qiancheng Xu, Tao Wang and Xuemei Qin contributed equally to this work. 'Department of Critical Care Medicine, The First Affiliated Hospital of Wannan Medical College (Yijishan Hospital of Wannan Medical College), No.2, West road of Zheshan, Jinghu District, Wuhu 241000, Anhui, China Full list of author information is available at the end of the article
}

observation study in three hospitals in Wuhu and Maanshan cities in Anhui Province.

From January 1 to April 2, 2020, 79 patients with coronavirus infection were screened. Ten was severe and all of them received early awake PP combined with HFNC treatment (Table 1). COVID-19 was diagnosed using sputum or throat swab determined by real-time reverse transcription polymerase chain reaction (RT-PCR) assay. The severity of disease was graded according to the Guidelines for the Diagnosis and Treatment of Novel Coronavirus (2019-nCoV) Infection by the National Health Commission (trial version 5). The target time of prone positioning is more than $16 \mathrm{~h}$ per day and can be appropriately shortened according to the patient's tolerance. Target $\mathrm{SpO}_{2}$ was more than $90 \%$ of adult nonpregnant patients (Fig. 1a).

All the severe patients, with $\mathrm{PF}<300 \mathrm{mmHg}$, developed mild respiratory alkalosis and no alkalemia at the beginning of HFNC treatment. After PP, compared with the baseline, the median $\mathrm{PaCO}_{2}$ increases slightly [32.3 (29.3-34.0) vs. 29.7 (28.0-32.0), $p<0.001]$. The median $\mathrm{PaO}_{2} / \mathrm{FiO} 2$ (PF) was elevated significantly after PP (Fig. 1b). None of the patients progressed to critical condition or needed endotracheal intubation.

When the condition of patients with severe coronavirus deteriorated rapidly and the mortality rate was unacceptably high [1], we then put forward a concept "reduce the proportion of severe COVID-19 conversion to critical illness," based on the experience summarized during the treatment of COVID-19 in Anhui, China. Early awake PP combined with HFNC therapy was one 


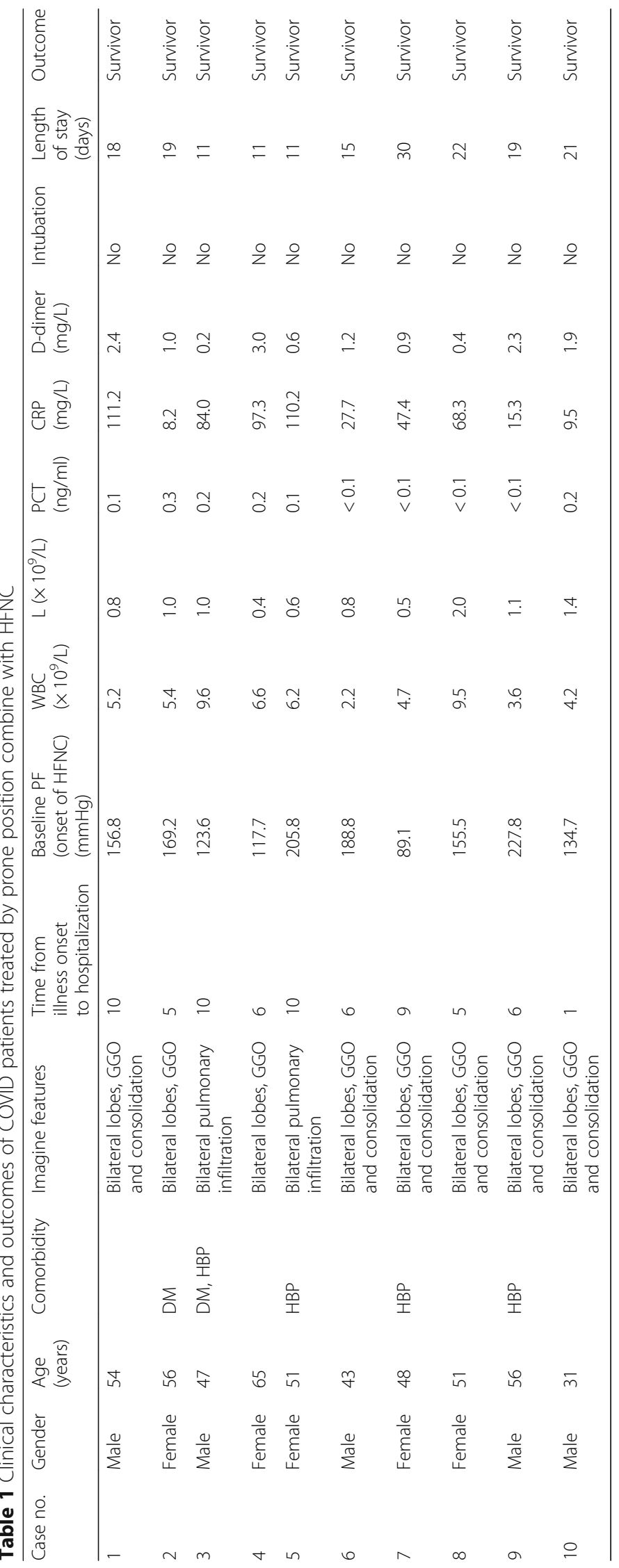



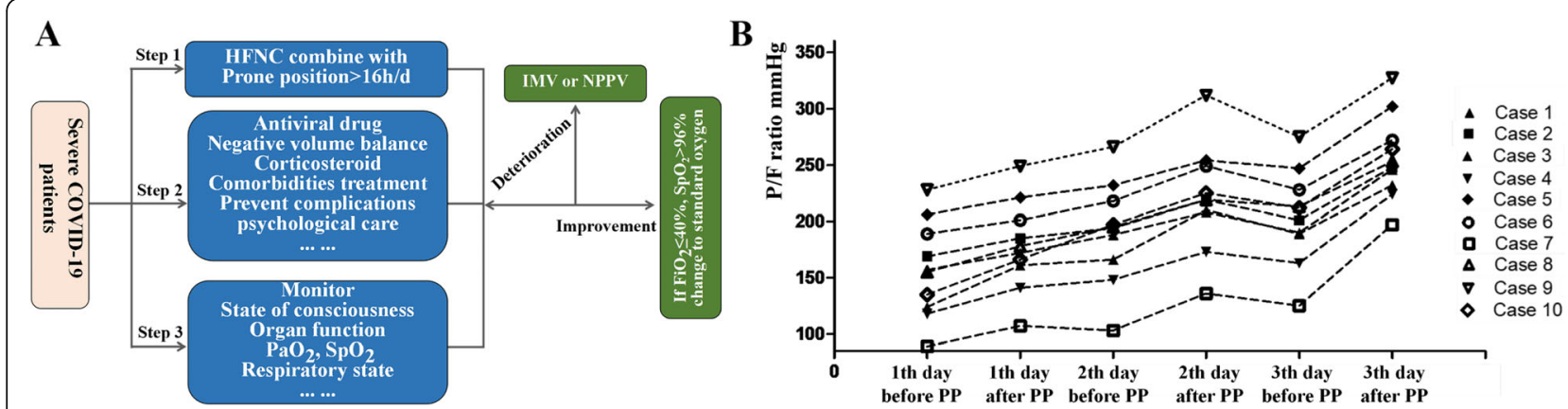

Fig. 1 a The protocol treatment of severe COVID-19 patients. b The change of $\mathrm{PaO}_{2} / \mathrm{FiO}_{2}$ in the first 3 days between onset of HFNC and 4-6 $\mathrm{h}$ after PP therapy in severe COVID-19 patients

of the most important strategies to avoid intubation and reduce the requirement for medical staff. A previous study reported that early application of PP with HFNC therapy, especially in patients with moderate ARDS, may help avoid intubation but not in the several ARDS group [5]. However, in non-infected severe ARDS patients with a $\mathrm{PF}<100 \mathrm{mmHg}, 5$ of 6 patients avoided intubation. In our research, the lowest $\mathrm{P} / \mathrm{F}$ ratio was $89 \mathrm{mmHg}$, and the patient was successfully discharged without invasive mechanical ventilation. The main reason for the patients' intolerance of PP is discomfort, anxiety [6], and the inability to change position. Our strategy was psychological care and a slight change of position every $2 \mathrm{~h}$.

Compared to non-invasive ventilation (NIV), patients felt more comfortable when using HFNC therapy, and the demand for medical staff was reduced. Awake PP combined with HFNC therapy could be used safely and effectively in severe COVID-19 patients, and it may reduce the conversion to critical illness and the need for tracheal intubation.

\section{Abbreviations}

COVID-19: Coronavirus disease 2019; HFNC: High-flow nasal cannula; PP: Prone position; PF: $\mathrm{PaO}_{2}, \mathrm{FiO}_{2}$; NIV: Non-invasive ventilation

\section{Acknowledgements}

Not applicable.

\section{Authors' contributions}

$\mathrm{Lu}$, the corresponding author, was responsible for the conceptualization of the study and the revision and approval of this manuscript. Xu, Qin, and Wang participated in the design, drafted the manuscript, and collected the data and were responsible for its accuracy. Zha helped to revise the manuscript. All authors contributed to the data analysis and interpretation. All authors read and approved the final manuscript.

\section{Funding}

This work was supported, in part, by the Anhui Provincial Special Project of Central Government Guiding Local Science and Technology Development of China (201907d07050001) and the special fund for coronavirus disease 2019 of Wuhu (no. 2020dx2-1 and 2020dx2-2).
Ethics approval and consent to participate

The study was approved by the First Affiliated Hospital of Wannan Medical College and participating institution ethics committees (approval number 2019-97). Written consent was obtained from each patient or his/her authorized representatives.

\section{Consent for publication}

All authors have approved the manuscript and its publication.

\section{Competing interests}

The authors have no competing interests.

\section{Author details}

${ }^{1}$ Department of Critical Care Medicine, The First Affiliated Hospital of Wannan Medical College (Yijishan Hospital of Wannan Medical College), No.2, West road of Zheshan, Jinghu District, Wuhu 241000, Anhui, China. ${ }^{2}$ Department of Critical Care Medicine, The Second People's Hospital of Wuhu, No 265, Jiuhua Road, Jinghu District, Wuhu 241000, Anhui, China. ${ }^{3}$ Institute of Infection and Global Health, University of Liverpool, Liverpool L69 7BE, UK.

Received: 27 April 2020 Accepted: 13 May 2020

Published online: 24 May 2020

\section{References}

1. Yang $X, Y u Y, X u$ J, Shu H, Xia J, Liu H, Wu Y, Zhang L, Yu Z, Fang M, et al. Clinical course and outcomes of critically ill patients with SARS-CoV-2 pneumonia in Wuhan, China: a single-centered, retrospective, observational study. Lancet Respir Med. 2020;8(5):475-81.

2. Bellani G, Laffey JG, Pham T, Fan E, Brochard L, Esteban A, Gattinoni L, van Haren F, Larsson A, McAuley DF, et al. Epidemiology, patterns of care, and mortality for patients with acute respiratory distress syndrome in intensive care units in 50 countries. JAMA. 2016;315(8):788-800.

3. Thille AW, Muller G, Gacouin A, Coudroy R, Decavele M, Sonneville R, Beloncle F, Girault C, Dangers L, Lautrette A, et al. Effect of postextubation high-flow nasal oxygen with noninvasive ventilation vs high-flow nasal oxygen alone on reintubation among patients at high risk of extubation failure: a randomized clinical trial. JAMA. 2019;322(15):1465-75.

4. Liu Q, Wang RS, Qu GQ, Wang YY, Liu P, Zhu YZ, Fei G, Ren L, Zhou YW, Liu L. Gross examination report of a COVID-19 death autopsy. Fa Yi Xue Za Zhi. 2020;36(1):21-3.

5. Ding $L$, Wang $L, M a W, H e ~ H$. Efficacy and safety of early prone positioning combined with HFNC or NIV in moderate to severe ARDS: a multi-center prospective cohort study. Crit Care. 2020;24(1):28.

6. Disilvio B, Young M, Gordon A, Malik K, Singh A, Cheema T. Complications and outcomes of acute respiratory distress syndrome. Crit Care Nurs Q. 2019;42(4):349-61

\section{Publisher's Note}

Springer Nature remains neutral with regard to jurisdictional claims in published maps and institutional affiliations. 